\title{
Gastritis aguda por Anisakis
}

\author{
Carmen Monroy Gómez ${ }^{\mathrm{a}}$, Agustín Santamaría Marín ${ }^{\mathrm{b}}$, Inés Clemente Toméa, \\ $\mathrm{M}^{\mathrm{a}}$ Vicenta Mayoralas Palomo ${ }^{\mathrm{b}}$.
}

\begin{abstract}
a Servicio de Medicina Interna. Hospital General Universitario de Ciudad Real. Ciudad Real (España).

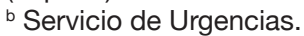
Hospital General Universitario de Ciudad Real. Ciudad Real (España).
\end{abstract}

Correspondencia:

Carmen Monroy Gómez.

Servicio de Medicina Interna.

Hospital General Universitario de Ciudad Real.

C/ Obispo General Torijo, s/n.

C.P. 13005 - Ciudad Real

(España).

Correo electrónico:

carmenmonroy82@hotmail.com

Recibido el 27 de enero de 2014.

Aceptado para su publicación el 8 de febrero de 2014.

\section{RESUMEN}

La anisakiasis es una enfermedad parasitaria que se adquiere al ingerir pescado crudo infestado por larvas de nematodos de la familia Anisakidae. Su diagnóstico es difícil, ya que la sintomatología es muy variable, pudiendo simular diversos cuadros clínicos. Por ello, es fundamental considerar los antecedentes epidemiológicos del paciente.

Palabras clave: Anisakis. Dolor Abdominal. Gastroscopia.

\section{ABSTRACT}

\section{Acute gastritis caused by Anisakis}

Anisakiasis is a parasitic disease caused by anisakid nematodes of the family Anisakidae. The disease is transmitted when infective larvae are ingested from raw fish. It is difficult to diagnose as the disease signs and symptoms may vary widely, producing different clinical pictures. For this reason it is essential to take into account the patient's epidemiological history.

Key words: Anisakis. Abdominal Pain. Gastroscopy.

\section{INTRODUCCIÓN}

La anisakiasis es una zoonosis causada por el consumo de la larva de Anisakis, presente con frecuencia en el pescado crudo o poco cocinado. El Anisakis es un nematodo tisular que pertenece a la familia Anisakidae. Se encuentra en el estómago e intestino de algunos mamíferos marinos como el delfín o la ballena. Existen 4 especies de este género, siendo el $A$. simplex el más frecuente.

Es una enfermedad de distribución mundial, aunque es más frecuente en los países que tradicionalmente consumen pescado crudo, como Japón y los países del norte de Europa. En España la prevalencia se sitúa entre el 5\% y el $56 \%$, aunque se cree que está infradiagnosticada ${ }^{1}$.

Las manifestaciones clínicas son muy variadas y dependen de los fenómenos de hipersensibilidad inmediata mediada por Ig E y del efecto local del parásito sobre el tubo digestivo².

Existen tres formas clínicas de presentación: gástrica, intestinal y extraintestinal (hígado, pulmón y peritoneo, entre otras) por migración de las larvas. En la forma gástrica la sintomatología es muy inespecífica; sin embargo, en la intestinal, el dolor abdominal, que es el síntoma más frecuente, en ocasiones es tan intenso que puede sugerir un abdomen agudo similar al de una apendicitis, peritonitis y obstrucción intestinal ${ }^{2,3}$.

\section{OBSERVACIONES CLÍNICAS}

Varón de 43 años sin antecedentes personales de interés que acude al servicio de urgencias por dolor abdominal de predominio en epigastrio y náuseas 


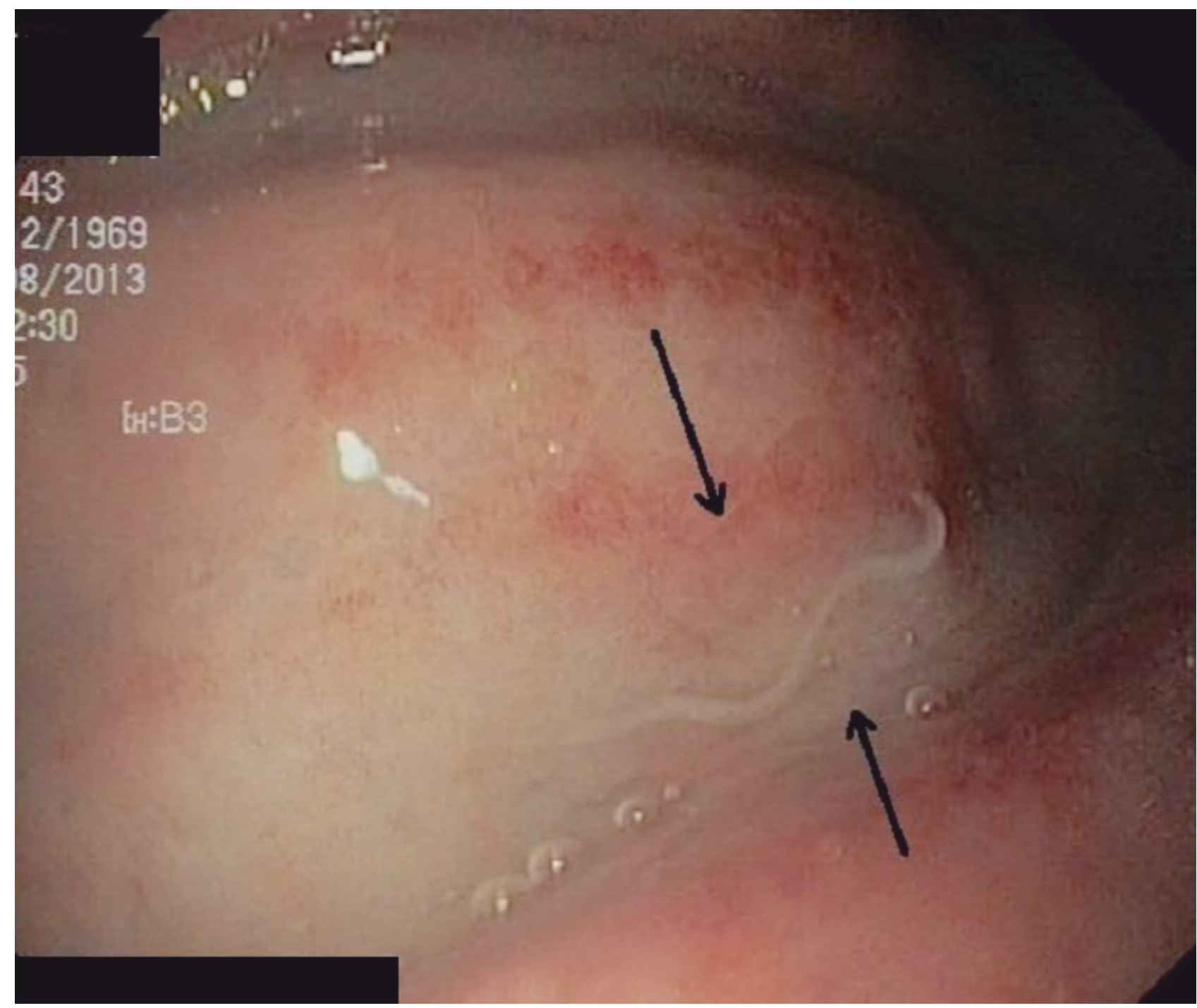

Figura 1. A nivel de cuerpo gástrico se observa un nematodo tisular de aproximadamente $2 \mathrm{~cm}$ de longitud, de color blanquecino, casi transparente, además de zonas eritematosas y erosiones superficiales.

de 4 días de evolución. En la exploración física el abdomen estaba distendido, doloroso de forma generalizada, con signos de peritonismo y ruidos hidroaéreos aumentados. La analítica fue normal. Se inició tratamiento analgésico y se realizó una ecografía abdominal en la que sólo se evidenció líquido libre peritoneal, motivo por el cual se realizó tomografía computerizada (TC) abdominal. En la TC abdominal se describía un engrosamiento difuso de la pared gástrica, aumento de densidad de la grasa perigástrica y líquido libre peritoneal. Tras estos hallazgos se reinterrogó al paciente, que refirió haber comido pescado crudo (boquerones en vinagre) el día previo al comienzo de los síntomas. Ante la sospecha de anisakiasis se realizó gastroscopia urgente (figura 1), en la que se observó a nivel de la curvatura mayor del cuerpo gástrico un parásito móvil compatible con Anisakis. Se realizó extracción endoscópica sin complicaciones y se envió a microbiología, que confirmó que se trataba de un Anisakis spp. La evolución clínica fue favorable, siendo dado de alta a las 24 horas de su ingreso en urgencias con omeprazol.

\section{COMENTARIOS}

La anisakiasis gástrica está producida por la acción local del parásito sobre el estómago, tras un periodo de latencia de 24-48 h. de la ingestión de pescado infestado ${ }^{1}$. Las formas gástricas representan el $60-70 \%$ de los casos 3 .

En el estómago, el parásito se fija a la mucosa, produciendo fenómenos irritativos locales que ocasio- 
nan dolor epigástrico intenso, náuseas, vómitos y febrícula a las pocas horas de la ingesta de pescado crudo. Puede acompañarse de rash cutáneo ${ }^{4}$. En algunos casos, aunque es menos frecuente, puede ser causa de hemorragia digestiva alta.

La analítica es muy inespecífica, siendo lo más habitual la leucocitosis con neutrofilia y la elevación de los reactantes de fase aguda. La eosinofilia periférica aparece en el $4-40 \%$ de los casos. Los hallazgos radiográficos tampoco son específicos. En la ecografía habitualmente se observa un engrosamiento de la pared del tramo afectado y líquido libre. Así pues, la endoscopia digestiva alta (EDA) será la que aporte el diagnóstico en la mayoría de las formas gastroduodenales mediante la visualización directa del parásito ${ }^{1-3}$. Sin embargo, hay ocasiones en las que éste no puede ser aislado, y tenemos que usar técnicas inmunológicas para realizar el diagnóstico definitivo ${ }^{1}$. Las más utilizadas son la determinación de lg $\mathrm{E}$ total, las inmunoglobulinas circulantes específicas y las proteínas catiónicas de los eosinófilos.

La EDA, además de ser diagnóstica es terapéutica, ya que permite la extracción del parásito con pinza mejorando rápidamente la sintomatología, como sucedió en nuestro caso.

Por lo tanto, ante un paciente con un cuadro clínico compatible y con el antecedente de la ingesta de pescado poco cocinado estaría indicada la realización de endoscopia urgente, ya que la extracción endoscópica es el tratamiento definitivo.

El tratamiento con albendazol es controvertido, aunque algunos estudios hablan de su eficacia ${ }^{5}$. Tampoco existe suficiencia evidencia en cuanto al tratamiento con inhibidores de la bomba de pro- tones. Hace años se consideró su utilidad basada en la observación clínica de que esta enfermedad rara vez se había descrito en pacientes gastrectomizados o en mayores de 60 años ${ }^{6}$, pero no hemos encontrado estudios recientes que apoyen este hecho.

En resumen, el dolor abdominal es una de las causas más frecuentes de consulta en los servicios de urgencias y es por ello, que el médico de atención primaria debe conocer las patologías que puedan requerir una actuación inmediata. La anisakiasis es una enfermedad con una incidencia creciente, por lo que debido a sus importantes repercusiones clínicas, debe ser sospechada e incluida en el diagnóstico diferencial de las enfermedades digestivas.

\section{BIBLIOGRAFÍA}

1. Henríquez Santana A, Villafruela Cives M. Anisakis: pasado, presente y futuro. Med Clin (Barc). 2009; 132 (10): 400-3.

2. González Quijada S, González Escudero R, Arias García L, Gil Martín AR, Vicente Serrano J, Corral Fernández E. Manifestaciones digestivas de la anisakiasis: descripción de 42 casos. Rev Clin Esp. 2005; 205 (7): 311-5.

3. López-Peñas D, Ramírez-Ortiz LM, Del Rosal-Palomeque R, López-Rubio F, Fernández-Crehuet R, Miño-Fugarolas G. Anisakiasis en España: una enfermedad creciente. Revisión. Gasteoenterol Hepatol 2000; 23 (6): 307-11.

4. Hochberg NS, Davidson HH. Anisakidosis: Perils of the deep. Clin Infect Dis. 2010; 51 (7): 806-12.

5. Kim SH, Park CW, Kim SK, Won S, Park WK, Kim HR et al. A case of anisakiasis invading the stomach and the colon at the same time after eating anchovies. Clin Endosc. 2013; 46 (3): 293-6.

6. Murakoa A, Suehiro I, Fujii M, Nagata K, Kusunoki H, Kumon $Y$ et al. Acute gastric anisakiasis: 28 cases during the last 10 years. Dig Dis Sci .1996; 41 (12): 2362-5. 